\title{
The experience of family caregivers in treatment of COVID-19 patients: a qualitative study
}

\author{
Maryam Nakhae ${ }^{1}$, Seyyed Abolfazl Vagharseyyedin¹, Gholamhossein Mahmoudirad ${ }^{1}$, \\ Hossein Shahdadi² \\ ${ }^{1}$ Department of Nursing, Faculty of Nursing and Midwifery, Birjand University of Medical Sciences, Birjand, Iran \\ ${ }^{2} \mathrm{PhD}$ student of nursing, Birjand University of Medical Sciences, Birjand, Iran
}

Neuropsychiatria i Neuropsychologia 2021; 16, 3-4: 131-137

Address for correspondence:

Gholamhossein Mahmoudirad, Assoc. Prof.

Department of Nursing

Faculty of Nursing and Midwifery

Birjand University of Medical Sciences

Birjand, Iran

e-mail: mahmoudirad@gmail.com

\begin{abstract}
Introduction: COVID-19 has drastic effects on both patients and their caregivers, so that they experience great problems. Hence, the present study was performed with the aim of explaining the home care experiences of patients with COVID-19.

Material and methods: This qualitative study was performed with the conventional content analysis approach and 16 primary caregivers of patients with COVID-19 who had undergone the acute phase of the disease were selected by the purposive sampling method. This study was conducted from 1 July 2020 to 1 March 2021. Data collection was performed by unstructured interviews, and data analysis was performed based on five steps of Graneheim and Lundman.

Results: From data analysis, 383 initial codes were extracted, which were obtained by continuous comparison and integration of subcategories of 6 main categories, i.e. fear, sincere care, quarantine atmosphere, paradoxical cyberspace, risk taking and positive care achievements.

Conclusions: Although home care was associated with problems and difficulties for caregivers, it ultimately brought caregivers a sense of inner satisfaction with this type of care and brought them positive results. Therefore, health professionals in such situations can provide the necessary training to reduce the difficulties of care and on the other hand, with proper planning, the ability of family members to care for patients and reduce the burden of care for medical staff.
\end{abstract}

Key words: care experience, home care, COVID-19.

\section{Introduction}

The new coronavirus disease 2019 (COVID-19) is an acute respiratory illness that is closely related to SARS coronavirus (Shigemura et al. 2020). In late December 2019, a new coronavirus triggered the outbreak of Wuhan pneumonia throughout China and has now posed major health threats to global public health. There are more than 182,383,450 patients with COVID-19 in the world with more than 3,948,969 deaths (Worldometer 2021). The disease has a range of symptoms including fever, cough, fatigue and muscle pain, shortness of breath, leukopenia, gastrointestinal problems and lung involvement (Duggan et al. 2021). Because of the increase in the number of patients infected with this virus, most hospitals have restrictions in admitting these patients. Hence, those who do not have a special need for hospital care should stay at home. Home care can have some advantages (Aminizadeh et al. 2021). In a study conducted by Barasteh et al. (2021) on the advantages of developing home health care in Iran, it was concluded that economic, social, and family advantages for patients, family and health system are among the benefits of home care, and home care strengthens solidarity in families, community and the health system and improves the quality of life of patients, community health and reduces health costs. Home care increases patient and family satisfaction and leads to an increase in the quality of services provided and a reduction in complications resulting from hospital processes (Raeisi et al. 2020). 
Caring for patients with COVID-19 has special conditions because this disease is a new and unknown disease for the medical staff, the patient and his family. Medical staff and family members have never experienced dealing with such patients. Caregivers of these patients are also at risk of being exposed to the virus and are concerned about infecting a family member. Al-Rabiaah's findings showed that perceived stress caused by the disease affects not only patients but also nurses, family members and other therapists (Al-Rabiaah et al. 2020).

These patients and those who are in contact with them should observe quarantine conditions to reduce the spread of the disease and reduce the risk of infecting others. Staying in quarantine exposes patients and their families to new conditions that are have different psychological and physical effects. When Cava et al. (2005) examined the experiences of quarantine patients with SARS in Toronto, they found that despite individual differences, they felt insecure, isolated, and struggling. They had to deal with psychological pressures and stresses.

However, the experiences of family caregivers of patients with COVID-19 and determining the problems related to this type of care lead to better planning to reduce stress on the patient and family members, and subsequently appropriate use of family members' abilities in caring for patients in certain situations. Due to the high workload of medical centers and the erosion of health care providers, home care will become more necessary than ever. Quantitative research does not have the necessary flexibility and depth to discover the experience of a phenomenon. For this reason, in order to deeply understand and describe the experiences of patient caregivers in an understandable way, qualitative studies are more suitable, especially content analysis, which is a good way to gain a deep and clear understanding of the phenomena. Hence, the present study was performed to investigate the home care experiences of patients with COVID-19.

\section{Material and methods}

In the present qualitative study, using the conventional method of a content analysis approach, home care experiences of patients with COVID-19 were investigated. This study was conducted from 1 July 2020 to 1 March 2021. In this study, 16 caregivers of patients with COVID-19, who played a key role in patient care and whose patients had passed the acute stage of the disease, had rich experience in the subject and the ability to communicate and the desire to participate in the study, were selected by purposive sampling. The process of selecting participants continued until data saturation, i.e. lack of access to new concepts and codes in the next interview, and according to the principle of maximum variation in sampling, it was attempted to involve caregivers in this study. Therefore, in terms of age, sex, level of education, social class, severity and duration of their disease, the patients were in different categories.

For data collection, in-depth unstructured interviews using open-ended questions were used focusing on the home care experience of patients with COVID-19 by phone, or face to face with suitable conditions (by considering the risk of disease transmission). At the beginning of the interview, questions were asked about demographic information such as education, marriage, etc., and then the interview was started using guiding questions such as "What does this disease mean to you?" and "What did you experience when looking after the patient?". The follow-up questions were asked based on the information provided by the participants to clarify the content of the study and in this regard they were asked questions such as "Can you explain more?" or "Can you give an example in this regard?". The duration of each interview was between 45 and 60 minutes depending on the desire and mental and physical condition of the participants and, if necessary, each participant was asked to be re-interviewed either by phone or face to face in suitable conditions (by considering the risk of transmission of the disease). Also, after each interview, the data were analyzed and the items that needed to be considered in the next interview were identified. It should be noted that in order to conduct a telephone interview with the supervisors, the necessary coordination was done about the time of the interview, and if the interview was to be held in person, the appropriate conditions for the interview location in terms of ventilation, comfort and convenience of participants were taken into account.

All interviews were recorded, typed, reviewed, coded, and immediately analyzed by the researcher, and in fact, data analysis was performed simultaneously and continuously with data collection. Data analysis was done based on five Graneheim, Lundman steps: 1) Writing the whole interview immediately after each interview (Transcribing); 2) Reading the whole text of the interview to gain a general understanding of its content and determining 
the units of meaning and codes (Meaning Units); 3) Abstracting semantic units and initial codes (Abstracting); 4) Classifying similar primary codes into more comprehensive classes (Sorting Codes); and 5) Determining the content hidden in the data (Formulating themes) (Mayring 2004; Speziale et al. 2011).

Criteria from Lincoln and Gouba were used to determine the credibility and reliability (Rigor) of this study (Lincoln and Guba 1986). In order to validate the findings, before conducting the interview, the researcher communicated with the participants several times (by phone or face to face) to gain the trust of the participants and create a suitable relationship and atmosphere for in-depth interviews. Also, the findings of the study were re-discussed with the participants (Member Check).

Confirmability of the results was also ensured by careful consideration in data collection and avoidance of any misuse and use of participants who could provide important information. Transferability of the results was ensured by using different demographic characteristics and different experiences and the study of all aspects of their behaviors, events and live experiences. Dependability was provided by sending a part of the interview text along with the relevant codes and the classes that appeared to several observers to review the analysis process and comment on their accuracy. Also, all activities and decisions, including data collection and analysis, were recorded separately and in the text in order to provide research for others if necessary.

In the present study it was attempted to explain the purpose and details of research methodology to the participants and to assure them that their identities would not be revealed during the research and dissemination of findings, and to maintain the anonymity of individuals, each one was assigned a number and people's quotes were reported based on these numbers, and if they did not want to continue at any stage of the research, they could be excluded from the study. In addition, the necessary permits for the study, including the permit of the ethics committee of Birjand University of Medical Sciences and written and informed consent, were obtained from the participants.

\section{Results}

The present study was done on 16 people who were primarily responsible for the home care of COVID-19 patients. Participants, of whom 10 were female and 6 were male, ranged in age from 20 to 60 years. In terms of educa- tion level, 2 people were illiterate, 3 people had elementary education, 4 people had secondary education, 4 people had high school diploma, 2 people had a bachelor degree and 1 had an M.Sc. degree. From data analysis, first 383 code were extracted, and by continuous comparison and merging, 6 subcategories of fear, sincere care, quarantine atmosphere, paradoxical cyberspace, risk taking, and positive care outcomes were extracted (Table 1).

\section{Fear}

One of the most important experiences of caregivers was the fear of infecting a family member with coronavirus, which was quoted in most of the reports. The ambiguity of the nature of the disease and the fear of the death of a family member caused fear of the disease. They feared that the disease was unknown and the possible adverse effects of the disease, and directly and indirectly stated that the nature of the disease had made it painful for any member of the family. A participant who was the wife of one of the patients stated in this regard: "I was very scared when I found out that my wife had COVID-19 because no one knew what the disease was like, what its complications were, what effects it had on the body. I was scared because I knew nothing" (37-year-old female participant).

Also, caregivers feared the possible death of any family member following coronavirus disease, and the possibility of death of the patient or other family members had become a night-

Table 1. Categories and subcategories of qualitative content analysis of interviews

\begin{tabular}{|c|c|c|}
\hline No. & Category & Subcategories \\
\hline \multirow[t]{2}{*}{1} & \multirow[t]{2}{*}{ Fear } & The ambiguous nature of the disease \\
\hline & & Fear of death \\
\hline \multirow[t]{2}{*}{2} & \multirow{2}{*}{$\begin{array}{l}\text { Sincere } \\
\text { care }\end{array}$} & Caring difficulties \\
\hline & & Providing the best possible care \\
\hline \multirow[t]{3}{*}{3} & \multirow{3}{*}{$\begin{array}{l}\text { Difficulties } \\
\text { of quaran- } \\
\text { tine }\end{array}$} & Quarantine difficulties \\
\hline & & Psychological trauma \\
\hline & & Social support \\
\hline \multirow[t]{2}{*}{4} & \multirow{2}{*}{$\begin{array}{l}\text { Paradoxical } \\
\text { cyberspace }\end{array}$} & Stressful cyberspace \\
\hline & & Helper cyberspace \\
\hline \multirow[t]{2}{*}{5} & \multirow[t]{2}{*}{ Risk taking } & Distrust in hospital care \\
\hline & & Preferring home care \\
\hline \multirow[t]{2}{*}{6} & \multirow{2}{*}{$\begin{array}{l}\text { Positive } \\
\text { care achie- } \\
\text { vements }\end{array}$} & Prefer home care \\
\hline & & Caring experiences \\
\hline
\end{tabular}


mare for them. They feared that if both of them died, their children would be left alone. In this regard, the wife of one of the patients who had three children stated, "Coronavirus was a scary nightmare for me. I always thought that if my wife or I died, what would happen to these children" (30-year-old female participant).

\section{Sincere care}

Caregivers of patients with COVID-19, despite experiencing a great deal of difficulties during care that made it difficult for them to care for patients, tried their best to provide the best possible care and ability. Most caregivers stated that during home care, they experienced suffering from loneliness, had concerns about deterioration of the patient, confusion in patient care, and lack of knowledge of the patient's required treatments. One of the participants said, "What bothered me the most was that I had to take care of my mother alone, there was no one to help me, and I was very worried about what I should do if my mother felt bad. I did not know what could be done to cure this disease" (27-year-old female participant).

However, they did their best to take care of their patients. They tried to give hope to their patient, to have sympathy, and to be by his side all the time. Despite knowing that they might have the disease themselves, they did not withhold any care from their patients. One caregiver whose general condition was not good compared to others said, "I used to suffer a lot whenever my father got sick, but I loved him so much that I did anything I could, I came to him, I gave him medicine with all his difficulties, I connected the ventilator, I cooked soup, and I did not even think for a moment that I might get this disease from my father, it did not matter to me at all" (40-year-old male participant).

\section{Quarantine atmosphere}

Caregivers felt obligated to comply with quarantine to prevent others from being infected. The conditions created during the quarantine made it very difficult for them during the quarantine due to restrictions on leaving home. On the other hand, these limitations had made them so tired and worn out that they felt tired without doing any physical activity. "I had a lot of problems during the quarantine, for example, I was always tired without doing anything", said one participant (40-year-old male participant).

Other difficulties of quarantine conditions for caregivers include psychological trauma, including a sense of meaninglessness in life, a sense of emptiness, and a sense of exclusion from society during quarantine. One participant stated: "I was mentally disturbed during the quarantine situation, I felt that life had become meaningless to me, I felt that I no longer had a place in society" (45-year-old male participant).

Social support was another factor in the quarantine situation, as other people played an important role during the quarantine period. Some of them, with their supportive presence, tried to be a good guide for caregivers to help patients. Some people around them kept in touch with them and asked how they were doing, trying to cheer them up and make them happy and reduce their pain and suffering. Interestingly, some of the participants said that the disease had caused them to know people who may not have been close to them before. Accordingly, one of the participants stated that "this situation made us know more about those around us, those who we did not think would reach us in these days (the period of the child's illness) helped a lot, called us by phone or cooked for us, or sometimes they would stand a little farther away to see us, which would make us happy" (40-year-old male participant).

On the other hand, according to the participants, some of the relatives also behaved inappropriately and unsupportively and caused feelings of hatred, dissatisfaction and annoyance for caregivers and patients. Some participants stated that those around them treated them inappropriately, ran away from them, tried to avoid facing them even after recovery, or looked at them with a contemptuous look, which caused them discomfort or depression. One participant said, "Those who claimed to be by your side were different in difficult times. Their attitudes, behaviors, and actions made us suffer more" (45-year-old female participant).

\section{Paradoxical cyberspace}

The spread of the coronavirus has made the use of cyberspace more prominent than ever. However, according to the observers, this space had paradoxical effects. In some cases, cyberspace was a stressor for caregivers, for example by announcing corona casualties, comments from different people or providing inappropriate information in this space, causing more concern to caregivers. They stated that the announcement of corona casualties in the world and in Iran, or the complications caused by the disease, which were spread by various virtual 
channels and in most cases were not accurate information, affected them and made them more worried, and even weakened their morale. "Cyberspace bothered me a lot. As soon as the news of corona deaths was announced, I was stressed and I thought for a moment that my mother might be like everyone. I was always thinking about losing my mother" (30-year-old female participant).

Despite this, cyberspace was able to help caregivers in some cases, so that through cyberspace and sometimes video communication, caregivers were able to communicate with those around them or their patient's physician who could not be seen in person due to quarantine, and as a suitable alternative, use it for human communication. Some caregivers used it to fill lonely times during quarantine, and a group who could not be present at their place of business used the space for virtual business assistance. One of the participants stated that "Since I could not see my relatives in this situation, I often tried to make video contact with them through this space, and this caused both me and my husband to feel better after talking to them" (30-year-old female participant). Another participant stated that "due to quarantine, our work and business was in great trouble, but this space was able to help us greatly, and because my husband had a free job, he was able to continue buying and selling" (38-year-old female participant).

\section{Risk taking}

Despite the dangers of home care, caregivers accepted these risks and preferred to care for their patient at home. Some participants were distrustful of hospital care. They stated that they were afraid of their patients being hospitalized because they believed that the hospital, especially for COVID-19 patients, would not receive the necessary care due to the high workload and the large number of hospitalized patients. In addition, the patient may suffer from nosocomial infections in addition to the disease he is suffering from, and on the other hand, these patients need psychological support and in the hospital, due to the high workload, these needs may not be addressed. One caregiver said, "I did not want to take my wife to the hospital at all. This is not only my experience. Everyone I heard who got infected and with whom I talked agreed that home care is much better than the hospital. There are so many patients in the hospital that the only thing that cannot be taken care of is the patient's mood, and the patient gets a nosocomial infection" (47-year-old male participant).

Despite the contagious and unknown nature of the disease, caregivers preferred to care for their patients at home because there were no hospital restrictions for patients and their caregivers at home. They stated that if one of their family members became infected again, they would still take care of them at home. In this regard, one of the participants said: "It was true that this disease was unknown and there was a possibility of infecting my children and even myself, but it was comfortable in my patient's house and he felt more relaxed" (30-year-old female participant).

\section{Positive care achievements}

Caring for patients with COVID-19 at home, despite all the problems, was able to achieve positive results, including satisfaction with care, while also allowing caregivers to gain good care experiences during this period. Caregivers felt inwardly satisfied that they were able to care for patients with COVID-19 at home, they felt happy that they could meet their patients' needs, and they found the patient's recovery to be their own comfort. One caregiver said, "As soon as I did something for my mother, who had worked hard for me for years, for example, giving her medicine or preparing food for her, she felt not satisfied that she was not in a good mood and needed me that I was not willing to exchange it for anything" (34-year-old female participant).

On the other hand, the point that most participants acknowledged was that despite all the difficulties of caring for patients with COVID-19 at home, during this time they were able to gain new experiences, endure the hardships more and learn always to be hopeful. During this time, they learned how to perform some care that they had not been able to do before. One of the caregivers said, "I learned a lot, for example, when my sister was coughing, I learned how to cure her cough, or I did not know how to anything at all because I had to do well in those circumstances. It made me learn to connect with my sister" (45-year-old female participant).

\section{Discussion}

The aim of this study was to gain a deeper and richer insight into the experience of family caregivers in caring for patients with COVID-19 and based on the findings of this study, fear, sincere care, quarantine atmosphere, paradoxical 
cyberspace, risk taking and positive care achievements were obtained as the main categories.

Fear was one of the main subjects extracted from the interviews of caregivers of patients with COVID-19. Caregivers cited the unknown illness and possible death of the patient or other family members following the spread of the disease as the main reasons for their fear. These findings are consistent with a study by Liu et al., which assessed the experiences of health care providers during the COVID-19 crisis. One of the experiences reported by health care providers in this study was that in the face of this unknown disease and unpredictable risks, health care providers were afraid of contracting the disease and were concerned about their families (Liu et al. 2020). Also, the findings of the study of Kalateh Sadati and her colleagues who examined the experiences of nurses from the outbreak of COVID-19 in Iran showed that the unknown disease caused more ambiguity in providing nursing services and nurses transferred the stress of the disease to their own family members (Kalateh Sadati et al. 2021).

Caregivers tried their best to use all the facilities available in the home to do their best to meet the needs of patients despite all the sufferings and difficulties in caring for patients. In the study of Galehdar et al. (2020), the results of which were in line with the present study, the nurses stated that in spite of the high workload and fatigue caused by it and the possibility of developing COVID-19, they took care of the patients.

The quarantine atmosphere made caregivers tired and the restrictions on leaving home made it difficult for them to make ends meet during quarantine, causing psychological damage such as meaningless living, feelings of emptiness, and feelings of psychological destruction. In this context, Brooks et al. (2020) stated that fatigue, boredom and anger are caused by staying in quarantine. Khodabakhshi-Koolaee (2020) stated that quarantine at home was associated with negative emotions and severe stress during the corona outbreak.

Relatives also played an important role in this situation, so that some of them appeared beyond expectations and contributed effectively to their pain and suffering, but others also behaved inappropriately with caregivers and patients and caused them discomfort. The study by Wester and Giesecke (2019) showed that the medical staff who went to West Africa for Ebola treatment were not worried, but after returning home they became very scared and even isolated from family, relatives and friends because they thought that they would make them infected.

Caregivers were reluctant to transfer their patients to the hospital, and they preferred this care by accepting the risks of home care. They believed that the hospital did not provide the necessary psychological support to their patients and that the high workload of nurses prevented them from taking care of their patients. However, the presence of others around the patient at home causes more encouragement and easier tolerance of the disease. As Liu stated that health care providers did not have the experience and knowledge to care for patients during the COVID-19 crisis, they tried hard to minimize complications, but patients needed a lot of care, and during this care, patients' families could not accompany them, which made it difficult to care for patients (Liu et al. 2020). Galehdar's study also showed that during COVID-19 disease, nursing errors were due to their high workload, nurses' protective coverings, and fear of becoming ill. There was an increase in disease and distance from patients (Galehdar et al. 2020). These cases made most caregivers want to take care of patients at home.

Home care had positive advantages for caregivers. In spite of the difficulties of caring for their patient, they were satisfied and happy that they were able to help them in this situation. Despite all the difficulties, caring for patients with COVID-19 at home gained care experiences for caregivers. As Farhadi et al. (2018) showed in their study that one of the most prominent positive consequences of caring for family caregivers was their personal growth, caregivers considered that such care had made them more mature (Netto et al. 2009) and stated that care had increased their self-awareness (Navab et al. 2012) and increased their understanding. The feeling of mastery or expertise in caring for some caregivers is beyond the mastery of new skills in care and gives them the feeling that they have acquired skills that they never imagined acquiring (Farhadi et al. 2018).

\section{Conclusions}

At first, caregivers feared that each member of the family would suffer from COVID-19 and possible death, while quarantine conditions were fraught with problems and suffering for them, making it difficult for them to care for patients. The use of cyberspace sometimes helped them and in some cases created problems for them. Despite all this, the caregivers used the available 
facilities to the best of their ability to heal their patients. Because of their distrust of hospital care in COVID-19 conditions, they preferred to take care of their patients at home, which made them happy. Their satisfaction with care was provided while gaining new experiences for them. Therefore, health professionals in such situations can provide the necessary training to reduce the difficulties of care and on the other hand, with proper planning, the ability of family members to care for patients and reduce the burden of care for medical staff.

It is worth mentioning that this study had its own limitations, including the fact that in most cases, face-to-face interviews were not possible due to the specific conditions of this disease, and most of the interviews were conducted by phone. On the other hand, the reluctance of some caregivers to talk about all aspects of caring for their patients in order to avoid patient discomfort sometimes caused the research team not to have all the experiences of these caregivers.

\section{Acknowledgments}

The authors consider it necessary to thank and appreciate all the participants in this study and the Vice Chancellor for Research of Birjand University of Medical Sciences with ethics code of IR.BMUS.REC.1399.186.

\section{Disclosure}

The authors declare no conflict of interest.

\section{References}

1. Al-Rabiaah A, Temsah MH, Al-Eyadhy AA, et al. Middle East Respiratory Syndrome-Corona Virus (MERS-CoV) associated stress among medical students at a university teaching hospital in Saudi Arabia. J Infect Public Health 2020; 13: 687-691.

2. Aminizadeh M, Saberinia A, Salahi S, et al. Quality of working life and organizational commitment of Iranian pre-hospital paramedic employees during the 2019 novel coronavirus outbreak. Int J Health Manag 2021; 1-9.

3. Barasteh S, Rassouli M, Karimirad MR, Ebadi A. Future challenges of nursing in health system of Iran. Front Public Health 2021; 9.

4. Brooks SK, Webster RK, Smith LE, et al. The psychological impact of quarantine and how to reduce it: rapid review of the evidence. Lancet 2020; 395: 912-920.

5. Cava MA, Fay KE, Beanlands HJ, et al. The experience of quarantine for individuals affected by SARS in Toronto. Public Health Nurs 2005; 22: 398-406.

6. Duggan NM, Ludy SM, Shannon BC, et al. Is novel coronavirus 2019 reinfection possible? Interpreting dynamic SARS-CoV-2 test results. Am J Emerg Med 2021; 39: e1256.e3.
7. Farhadi A, Noroozian M, Mohammadi F, et al. Positive experiences of caregiving in family caregivers of older adults with dementia: a content analysis study. ISMJ 2018; 21: 319-334.

8. Galehdar N, Kamran A, Toulabi T, Heydari H. Exploring nurses' experiences of psychological distress during care of patients with COVID-19: a qualitative study. BMC Psychiatry 2020; 20: 489.

9. Kalateh Sadati A, Zarei L, Shahabi S, et al. Nursing experiences of COVID 19 outbreak in Iran: a qualitative study. Nurs Open 2021; 8: 72-79.

10. Khodabakhshi-KoolaeeA.Livinginhomequarantine: analyzing psychological experiences of college students during Covid-19 pandemic. J Military Med 2020; 22: 130138.

11. Lincoln YS, Guba EG. But is it rigorous? Trustworthiness and authenticity in naturalistic evaluation. New Directions for Program Evaluation 1986; 73-84.

12. Liu Q, Luo D, Haase JE, et al. The experiences of health-care providers during the COVID-19 crisis in China: a qualitative study. Lancet Glob Health 2020; 8: e790-e798.

13. Mayring P. Qualitative content analysis. A companion to qualitative research 2004; 1: 159-176.

14. Navab E, Negarandeh R, Peyrovi H. Lived experiences of Iranian family member caregivers of persons with Alzheimer's disease: caring as 'captured in the whirlpool of time'. J Clin Nurs 2012; 21: 1078-1086.

15. Netto NR, Jenny GYN, Philip YLK. Growing and gaining through caring for a loved one with dementia. Dementia 2009; 8: 245-261.

16. Raeisi A, Tabrizi JS, Gouya MM. IR of Iran national mobilization against COVID-19 epidemic. Arch Iran Med 2020; 23: 216-219.

17. Shigemura J, Ursano RJ, Morganstein JC, et al. Public responses to the novel 2019 coronavirus (2019-nCoV) in Japan: mental health consequences and target populations. Psychiatry Clin Neurosci 2020; 74: 281-282.

18. Speziale HS, Streubert HJ, Carpenter DR. Qualitative research in nursing: Advancing the humanistic imperative. Lippincott Williams \& Wilkins 2011.

19. Wester M, Giesecke J. Ebola and healthcare worker stigma. Scand J Public Health 2019; 47: 99-104.

20. Worldometer, 2021. Coronavirus cases [Online]. Available: https://www.worldometers.info/coronavirus/. 